\title{
Cation and anion transport through hydrophilic pores in lipid bilayers
}

\author{
Senthil K. Kandasamy ${ }^{\text {a) }}$ and Ronald G. Larson ${ }^{\text {b) }}$ \\ Chemical Engineering Department, The University of Michigan, Ann Arbor, Michigan 48109
}

(Received 23 March 2006; accepted 1 June 2006; published online 17 August 2006)

\begin{abstract}
To understand the origin of transmembrane potentials, formation of transient pores, and the movement of anions and cations across lipid membranes, we have performed systematic atomistic molecular dynamics simulations of palmitoyl-oleoyl-phosphatidylcholine (POPC) lipids. A double bilayer setup was employed and different transmembrane potentials were generated by varying the anion $\left(\mathrm{Cl}^{-}\right)$and cation $\left(\mathrm{Na}^{+}\right)$concentrations in the two water compartments. A transmembrane potential of $\sim 350 \mathrm{mV}$ was thereby generated per bilayer for a unit charge imbalance. For transmembrane potential differences of up to $\sim 1.4 \mathrm{~V}$, the bilayers were stable, over the time scale of the simulations (10-50 ns). At larger imposed potential differences, one of the two bilayers breaks down through formation of a water pore, leading to both anion and cation translocations through the pore. The anions typically have a short residence time inside the pore, while the cations show a wider range of residence times depending on whether they bind to a lipid molecule or not. Over the time scale of the simulations, we do not observe the discharge of the entire potential difference, nor do we observe pore closing, although we observe that the size of the pore decreases as more ions translocate. We also observed a rare lipid flip-flop, in which a lipid molecule translocated from one bilayer leaflet to the opposite leaflet, assisted by the water pore. (C) 2006 American Institute of Physics. [DOI: 10.1063/1.2217737]
\end{abstract}

\section{INTRODUCTION}

Most biological membranes have a transmembrane potential difference that affects the transport of various small and large molecules across the membrane. The potential difference arises primarily because of the difference in the distribution of charges between the inside and the outside of the cell. While molecular dynamics simulations of lipid bilayers have advanced tremendously in the past decade, explicit treatment of transmembrane potential difference has not been possible until recently. In most molecular dynamics simulations of lipid bilayers, a single lipid bilayer is simulated, with water layers on either side of the bilayer. Since periodic boundary conditions are commonly employed, the water layers are connected and hence, unlike cellular membranes, there is no inside or outside to the simulated bilayer. Thus, an explicit transmembrane potential difference cannot be imposed in simulations using a single bilayer and periodic boundary conditions. This has been recently overcome by simulating double bilayer, ${ }^{1,2}$ which has two lipid bilayers and two distinct water compartments. By changing the number of charges in the two water compartments, which is achieved by varying the anion and cation concentrations, a transmembrane potential can be explicitly simulated. However, simulating such systems is computationally more expensive because of the resulting twofold increase in the number of atoms.

There are three significant contributors to the transmembrane potential: (a) charge imbalance between the inside and outside of the membrane, (b) lipid head group dipole orien-

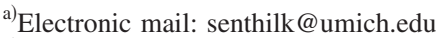

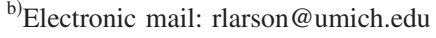

tation, and (c) ordering of the water molecules adjacent to the lipid head groups. ${ }^{3}$ In the presence of large transmembrane potentials induced by external electric fields, water pores can form in lipid bilayers. ${ }^{4}$ This has also been shown in simulations recently using an external electric field. ${ }^{5} \mathrm{~A}$ more recent study has shown that such water pores can spontaneously form in a double bilayer setup, with a sufficiently large transmembrane potential. ${ }^{6}$ In that study, pore formation and cation transport were observed on time scales of $\sim 10 \mathrm{~ns}$. Other studies with a similar double layer setup, but with an implicit membrane representation, have rigorously analyzed both anion and cation transports. 7,8

In this manuscript, by employing the double bilayer setup with atomic resolution, similar to earlier studies, ${ }^{1,2,6}$ we systematically investigate a wide range of transmembrane potentials, pore formation, and ion tranlocation in palmitoyloleoyl-phosphatidylcholine (POPC) lipid bilayers. In the study by Gurtovenko and Vattulainen, ${ }^{6}$ only cation transport was observed due to the nature of the ion imbalance. In this work, the transmembrane potential is generated by gradients in both the anion and cation concentrations, enabling us to study pore formation and subsequent anion and cation translocations across the bilayer. This is a first step towards the understanding of more challenging processes such as small molecule, drug, DNA, and peptide translocation across biological membranes.

\section{METHODS}

We used the GROMACS suite for all our simulations., 9 The double bilayer system was constructed by duplicating a preequilibrated single bilayer along the bilayer normal direction. The single bilayer consisted of 128 POPC lipids (64 per 


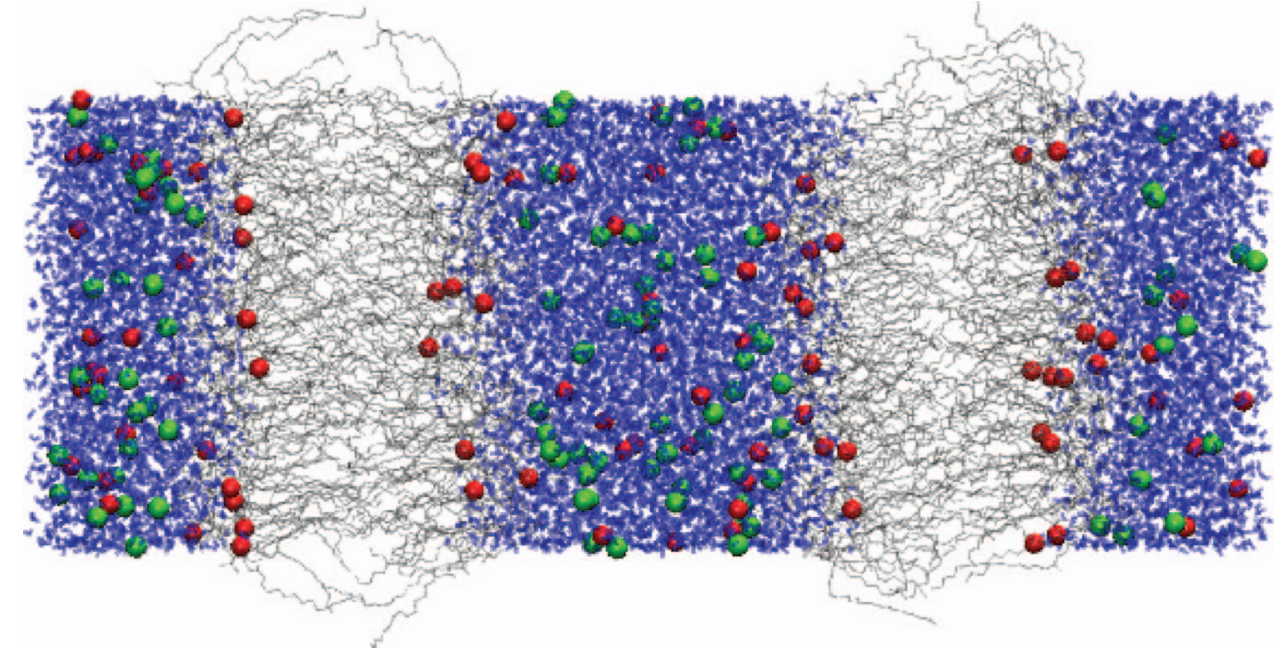

FIG. 1. (Color) A snapshot of the double bilayer setup. Periodic boundary conditions are applied in all three directions. The $\mathrm{Na}^{+}$ions are shown as red spheres and the $\mathrm{Cl}^{-}$ions are shown as green spheres. Transbilayer potential differences can be generated by varying the relative concentrations of $\mathrm{Na}^{+}$and $\mathrm{Cl}^{-}$ions in the two water compartments. leaflet) and $\sim 5000$ water molecules. The double bilayer consisted of 256 lipids (128 lipids per bilayer; 64 lipids per leaflet) and $\sim 10000$ water molecules; refer to Fig. 1 . The number of water molecules was chosen so that the thickness of the water layer separating the bilayers was larger than the region of expected salt-induced water ordering near the lipid head groups. The system dimensions were roughly 6.2 $\times 6.2 \times 16 \mathrm{~nm}^{3}$. For convenience, the water compartments will be referred to as the inner and outer compartments. $\mathrm{Na}^{+}$ and $\mathrm{Cl}^{-}$ions were the charged species used to generate the charge imbalance. In Table I, we list all 23 simulations performed in this study. We first simulated the double bilayer system with no ions for $50 \mathrm{~ns}$ (labeled D000-0). Then, we took the coordinates from this simulation after $20 \mathrm{~ns}$ and added one $\mathrm{Na}^{+}$ion to the outer water compartment and one $\mathrm{Cl}^{-}$ion to the inner water compartment, by replacing one water molecule per ion. While the net charge difference is thus two charge units, since there are two bilayers in the simulation, this generates a single unit charge imbalance across each bilayer in the double bilayer system. Then, we added an additional $\mathrm{Na}^{+}$ion to the outer bilayer and an additional $\mathrm{Cl}^{-}$ion to the inner bilayer. Similarly, we generated systems with a charge imbalance of up to $10 e$ per bilayer. These systems were labeled D000-1 to D000-10 and were simulated for at least $10 \mathrm{~ns}$ each. The $\mathrm{Na}^{+}$and $\mathrm{Cl}^{-}$ions were inserted randomly in the respective water compartments. Then we added $45 \mathrm{Na}^{+}$and $45 \mathrm{Cl}^{-}$ions to both compartments (total of 180 ions) to the simulation D000-0 at $20 \mathrm{~ns}$.

TABLE I. Transmembrane potential in different simulations.

\begin{tabular}{ccccccc}
\hline \hline & Label & $\begin{array}{c}\text { Inner } \\
\mathrm{Na}^{+} / \mathrm{Cl}^{-}\end{array}$ & $\begin{array}{c}\text { Outer } \\
\mathrm{Na}^{+} / \mathrm{Cl}^{-}\end{array}$ & $\begin{array}{c}\text { Time } \\
(\mathrm{ns})\end{array}$ & $\begin{array}{c}\text { Charge } \\
\text { imbalance }\end{array}$ & $\begin{array}{c}\text { Transmembrane } \\
\text { potential }(\mathrm{mV})\end{array}$ \\
\hline 1 & D000-0 & $0 / 0$ & $0 / 0$ & 50 & 0 & 0 \\
2 & D000-1 & $0 / 1$ & $1 / 0$ & 10 & 1 & 360 \\
3 & D000-2 & $0 / 2$ & $2 / 0$ & 10 & 2 & 770 \\
4 & D000-3 & $0 / 3$ & $3 / 0$ & 10 & 3 & 1150 \\
5 & D000-4 & $0 / 4$ & $4 / 0$ & 10 & 4 & 1400 \\
6 & D000-5 & $0 / 5$ & $5 / 0$ & 30 & 5 & $1750^{\mathrm{a}}$ \\
7 & D000-6 & $0 / 6$ & $6 / 0$ & 10 & 6 & $2130^{\mathrm{a}}$ \\
8 & D000-7 & $0 / 7$ & $7 / 0$ & 10 & 7 & $2520^{\mathrm{a}}$ \\
9 & D000-8 & $0 / 8$ & $8 / 0$ & 10 & 8 & $2810^{\mathrm{a}}$ \\
10 & D000-9 & $0 / 9$ & $9 / 0$ & 10 & 9 & $3100^{\mathrm{a}}$ \\
11 & D000-10 & $0 / 10$ & $10 / 0$ & 10 & 10 & $3390^{\mathrm{a}}$ \\
12 & D500-0 & $45 / 45$ & $45 / 45$ & 50 & 0 & 0 \\
13 & D500-1 & $45 / 46$ & $46 / 45$ & 50 & 1 & 350 \\
14 & D500-2 & $45 / 47$ & $47 / 45$ & 50 & 2 & 1030 \\
15 & D500-3 & $45 / 48$ & $48 / 45$ & 50 & 3 & 1370 \\
16 & D500-4 & $45 / 49$ & $49 / 45$ & 20 & 4 & $1720^{\mathrm{a}}$ \\
17 & D500-5 & $45 / 50$ & $50 / 45$ & 30 & 5 & $2220^{\mathrm{a}}$ \\
18 & D500-6 & $45 / 51$ & $51 / 45$ & 20 & 6 & $2490^{\mathrm{a}}$ \\
19 & D500-7 & $45 / 52$ & $52 / 45$ & 10 & 7 & $2800^{\mathrm{a}}$ \\
20 & D500-8 & $45 / 53$ & $53 / 45$ & 10 & 8 & $3070^{\mathrm{a}}$ \\
21 & D500-9 & $45 / 54$ & $54 / 45$ & 10 & 9 & $3430^{\mathrm{a}}$ \\
22 & D500-10 & $45 / 55$ & $55 / 45$ & 10 & 10 & 90 \\
23 & D500-D000 & $45 / 45$ & $0 / 0$ & 50 & 0 & \\
\hline \hline
\end{tabular}

${ }^{\mathrm{a}}$ These values were averaged from the beginning of the simulation to the time when the pore was formed. 
This corresponds to a concentration of $\sim 500 \mathrm{mM} \mathrm{NaCl}$ in each compartment. Here, the ions were added at electrostatically favorable locations by replacing one water molecule per ion. This simulation was labeled D500-0 and was simulated for 50 ns. A snapshot of this system is shown in Fig. 1. Red spheres are $\mathrm{Na}^{+}$ions and green spheres are $\mathrm{Cl}^{-}$ions. Then an additional $\mathrm{Na}^{+}$ion was added to the outer compartment and an additional $\mathrm{Cl}^{-}$ion added to the inner compartment to the coordinates at the end of this simulation. This generates a unit charge imbalance across each bilayer in addition to the balanced electrolyte. In a similar fashion, we generated charge imbalances of up to $10 e$ per bilayer. These simulations were labeled D500-1 to D500-10. Simulations D500-1, D500-2, D500-3, and D500-4 were run for $50 \mathrm{~ns}$ each while the other simulations were carried out for at least $10 \mathrm{~ns}$ each. Finally, taking the coordinates after $20 \mathrm{~ns}$ of simulation D000-0, we added $45 \mathrm{Na}^{+}$and $45 \mathrm{Cl}^{-}$ions to the outer water compartment and no ions to the inner compartment. This simulation was labeled D500-D000 and was simulated for $50 \mathrm{~ns}$. These 23 simulations provide us with a wide range of salt concentrations and ion imbalances.

We used the well-validated lipid force field by Berger $e t$ $a l .{ }^{11}$ that has been used in several bilayer studies. ${ }^{2,6,12-14}$ The simple point charge (SPC) model was used for water. The choice of parameters for ions can significantly affect the structural and dynamical properties of interest. ${ }^{15}$ We used the default ion parameters in the GROMACS force field, which has been used by other authors. ${ }^{2,6}$ All simulations were performed in the NPT ensemble. The temperature was maintained at $310 \mathrm{~K}$ by coupling the lipids and the rest of the system separately to a Berendsen thermostat with a coupling constant of $0.1 \mathrm{ps}$. The pressure was semi-isotropically coupled to a Berendsen barostat at $1 \mathrm{~atm}$ pressure with a coupling constant of 1 ps. Short-range interactions used a cutoff of $1.2 \mathrm{~nm}$. Long-range electrostatic interactions, which are extremely important in systems with many charges, ${ }^{16}$ were calculated using the particle mesh Ewald algorithm ${ }^{17}$ with tin-foil boundary conditions. Bonds were constrained using the LINCS algorithm. ${ }^{18}$ Coordinates were saved every picosecond and were used in subsequent analysis.

\section{RESULTS AND DISCUSSION}

\section{Transmembrane potential}

We calculated the potential profile of the systems by twice integrating the charge density along the axis parallel to the bilayer normal, averaged over both bilayers. ${ }^{2}$ The potential profile is shown in Fig. 2, left, for some of the simulated systems (D500-0 to D500-4). A scaled phosphorus density is shown as dotted lines for reference along the bilayer normal. The transmembrane potential, which is zero for D500-0, increases systematically as the charge imbalance increases. The potential was averaged over the last $20 \mathrm{~ns}$ for simulations D500-0, D500-1, D500-2, D500-3, and D500-4. The transmembrane potential increases by $\sim 350 \mathrm{mV}$ per bilayer with each additional unit charge imbalance. The magnitude of this potential is comparable to that obtained by a study which used a similar force field on a different $\operatorname{lipid}^{2}$ and yet

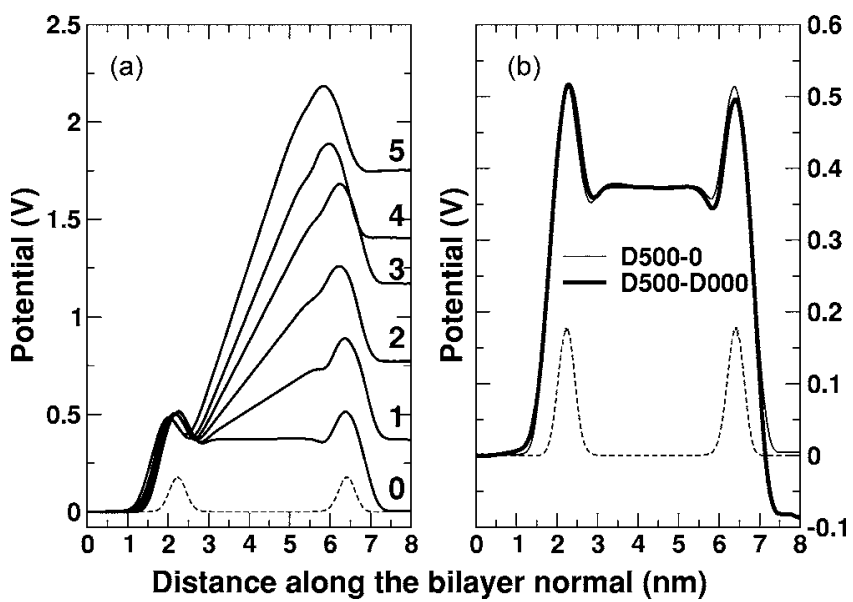

FIG. 2. (a) Potential profiles in the direction normal to the bilayer, for six simulations at $500 \mathrm{mM}$ salt. The thin dashed line is the arbitrarily scaled density of the lipid phosphorus atoms, shown for reference. The number on the right of each curve is the charge imbalance per bilayer, which is induced by varying the number of anions and cations in the two water compartments. A transbilayer potential difference of $\sim 350 \mathrm{mV}$ is generated for each additional charge imbalance. (b) Potential profiles for simulations D500-0 and D500-D000, which, respectively, are simulations with electrolyte in both water compartments and only in the outer compartment, both with no charge imbalance. A small transmembrane potential difference of $\sim 90 \mathrm{mV}$ is observed when the salt concentrations in the two water compartments are different, even though both the compartments are electrically neutral. The thin dashed line is the arbitrarily scaled density of the lipid phosphorus atoms, shown for reference.

another study which used different force fields for POPC lipids. ${ }^{1}$ This value is also close to estimated analytical values, as shown in the work by Sachs et al. ${ }^{1}$ We observe similar trends for systems at $0 M$ salt (D000-1 to D000-4), with a transmembrane potential of $\sim 350 \mathrm{mV}$ per leaflet for each additional charge imbalance. All systems with a charge imbalance of $4 e$ or less at $500 \mathrm{mM}$ salt were stable for times up to $50 \mathrm{~ns}$. The transmembrane potential differences are tabulated in Table I. In Fig. 2 right, we show the transmembrane potential profiles from simulations D500-0 and D500-D000. Both the water compartments are electrically neutral in the latter simulation, but there is a difference in the salt concentration between the two compartments. This induces a transmembrane potential ${ }^{2}$ of $\sim 90 \mathrm{mV}$ across the bilayer. The origin of this transmembrane potential lies in the differences in the ordering of the water near the interface and the phosphorus-nitrogen $(\mathrm{P}-\mathrm{N})$ lipid head group vector orientation on either side of the bilayer.

\section{Water dipole orientation}

In Fig. 3, we show the water molecule orientation near the lipid head group region. ${ }^{19}$ The cosine of the average orientation of the water dipole relative to the bilayer normal direction is shown. In this figure, we only show the water orientation in the inner water compartment. The outer water compartment is not shown. The scaled lipid phosphorus density is shown as a dashed line for reference and the vertical dotted line represents the approximate center of the water compartment. In D000-0 and D500-D000, there is no salt in this inner compartment. For each monolayer leaflet, the cosine of the average water dipole orientation shows a single 


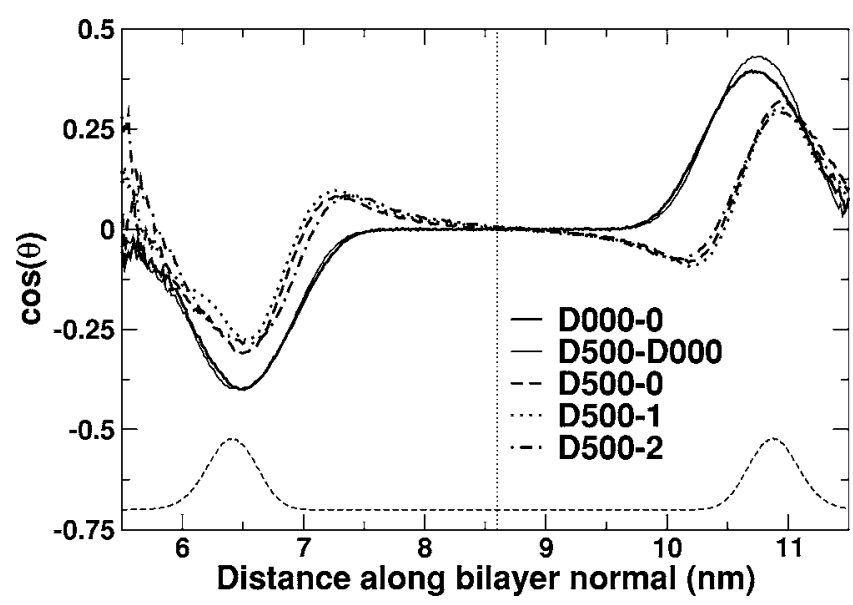

FIG. 3. The cosine of the average orientation of the water dipole relative to the bilayer normal. Water ordering is different at different salt concentrations.

peak (or dip) at approximately the location of the average phosphorus atom. However, for D500-0, D500-1, and D500-2, where there is salt present in both compartments, water is less ordered at the peak, the peak is shifted, and there is a second smaller dip, showing the effect of salt on the water ordering near the bilayer interface. The ordering of water is not affected significantly on adding additional $\mathrm{Cl}^{-}$ ions to the inner compartment (and $\mathrm{Na}^{+}$ions to the outer compartment), which leads to the transmembrane potential difference.

\section{Head group tilt}

The average orientation of the $\mathrm{P}-\mathrm{N}$ dipole relative to the bilayer normal has been shown to change with salt concentration. ${ }^{19-23}$ Earlier studies have shown that the average $\mathrm{P}-\mathrm{N}$ vector rotates more towards the bilayer normal with increasing salt concentration, which is related to the reduction in the average area available per lipid due to the $\mathrm{Na}^{+}$ ions binding to the lipid head groups. ${ }^{22}$ In Fig. 4, we show the distribution of the $\mathrm{P}-\mathrm{N}$ tilt angles for some of the simu-

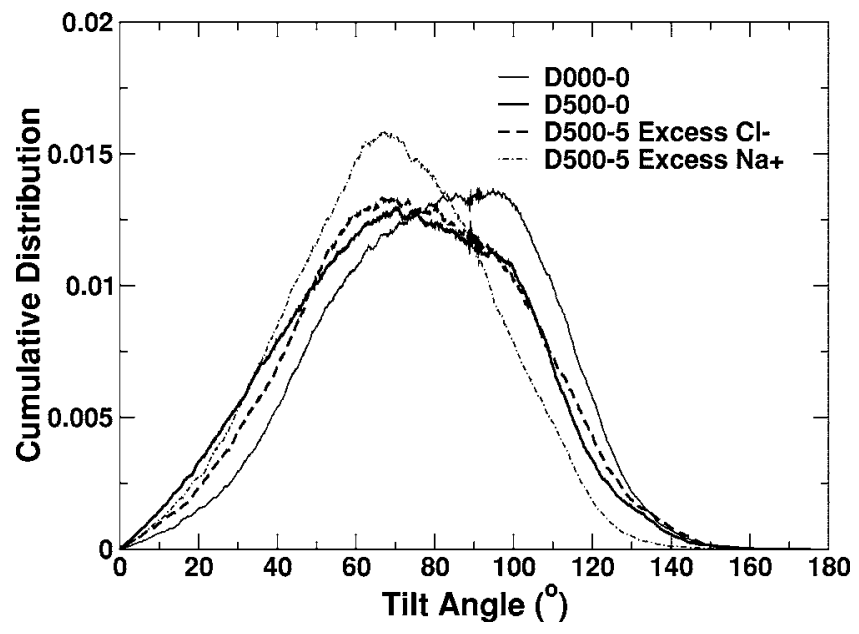

FIG. 4. Distribution of the average lipid head group $(\mathrm{P}-\mathrm{N})$ tilt angle relative to the bilayer normal. The average tilt angle decreases as the salt concentration is increased. In the presence of excess $\mathrm{Na}^{+}$ions, the tilt angle distribution is narrower. lations. We observe a reduction in the average tilt angle from $\sim 80^{\circ}$ to $\sim 70^{\circ}$ relative to the bilayer normal as the salt concentration is increased from 0 to $500 \mathrm{mM}$ (D000-0 and D500-0). As additional chloride ions are added to the inner compartment and $\mathrm{Na}^{+}$ions to the outer compartment (D5005 ), the average $\mathrm{P}-\mathrm{N}$ vector orientation in the inner compartment, which has excess $\mathrm{Cl}^{-}$ions, increases to $\sim 73^{\circ}$ and that of the outer compartment, which has excess $\mathrm{Na}^{+}$ions decreases further to $\sim 65^{\circ}$, with a narrower distribution. The same qualitative trend is observed for D500-1, D500-2, and D500-3, and D500-4, but to a smaller extent.

In each water compartment, a certain fraction of the $\mathrm{Na}^{+}$ ions are "bound" to the lipid head groups while the rest of the $\mathrm{Na}^{+}$ions are in the water phase. ${ }^{2,22}$ In the absence of an explicit charge imbalance between the two compartments (D500-0), the $\mathrm{Na}^{+}$ions in both water compartments behave similarly. However, with increasing charge imbalance, the fraction of bound $\mathrm{Na}^{+}$ions in both compartments increases, more so in the compartment with excess $\mathrm{Na}^{+}$ions and less in the compartment with excess $\mathrm{Cl}^{-}$ions. For example, in the last $10 \mathrm{~ns}$ of simulation D500-0, 40\% of the $\mathrm{Na}^{+}$ions are bound, while for simulation D500-5, $\sim 60 \%$ of $\mathrm{Na}^{+}$ions are bound in the compartment with excess $\mathrm{Na}^{+}$and $\sim 50 \%$ of the $\mathrm{Na}^{+}$ions are bound in the compartment with excess $\mathrm{Cl}^{-}$. Here, we consider a $\mathrm{Na}^{+}$ion to be bound if it is at distance of $<3 \AA$ from any lipid oxygen. Thus, the charge imbalance across the lipid bilayers causes the $\mathrm{Na}^{+}$ions to accumulate near the lipid-water interface, leading to large changes in the orientation of the $\mathrm{P}-\mathrm{N}$ dipole vector. Although the changes in the orientation of the water dipoles and the lipid head group dipoles do affect the transmembrane potential, the largest contribution to the transmembrane potential is still from the direct effect of the charge imbalance itself.

\section{Pore formation}

In systems with charge imbalances of $5 e$ or more, dielectric breakdown occurs, leading to the formation of pores in one of the two bilayers. This phenomenon was observed recently in dimyristoyl-phosphatidylcholine (DMPC) bilayers. ${ }^{6}$ The mechanism of pore formation is identical to that in the presence of an external electric field. ${ }^{5}$ Some water molecules near the head group region of the bilayer leaflets start aligning their dipoles, which enables deep penetration of water molecules into the hydrophobic core. Eventually, a single file of water passes across to the other side, which in turn leads to a rapid expansion of the pore, with some lipid head groups entering the hydrophobic core from both sides, lining the water pore. Once the pore is formed, both anions and cations pass through the pore to reduce the charge imbalance. In Fig. 5 , we show snapshots at three different times for the simulation D500-9. In Fig. 5(a) ( $\sim 1.5 \mathrm{~ns})$, the early stages of the pore formation are shown. Water molecules within a distance of $\sim 8 \AA$ of the bilayer center are highlighted. In Fig. 5(b) $(\sim 2.5 \mathrm{~ns})$, the pore is shown when it is the largest. We can see some $\mathrm{Na}^{+}$(red) and $\mathrm{Cl}^{-}$(green) ions translocating through the pore. In Fig. 5(c) $(\sim 10 \mathrm{~ns})$, after some ions have translocated, the pore is smaller than at $2.5 \mathrm{~ns}$. In Fig. 5(d), we show a close-up of the water molecules in the pore region 


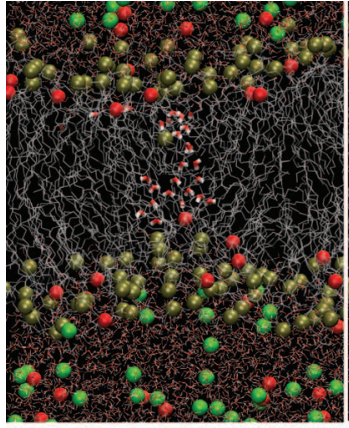

(a)

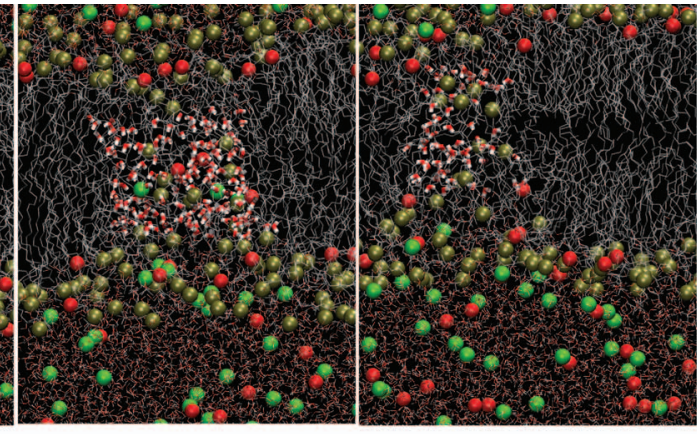

(b) (c)

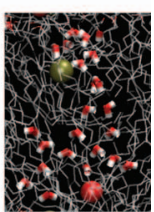

(d)
FIG. 5. (Color) [(a)-(c)] Snapshots of the pore region at $1.5,2.5$, and $10 \mathrm{~ns}$ of simulation D500-9. The lipids are shown as white lines. The water molecules in the pore region are shown as red and white bonds. The lipid phosphorus atoms, $\mathrm{Na}^{+}$ions, and $\mathrm{Cl}^{-}$ions are shown as gold, red, and green spheres, respectively. (d) A closer look at the water molecules at $1.5 \mathrm{~ns}$. This is during the early stages of pore formation and the water dipoles are all oriented in the direction of the bilayer normal. at $1.5 \mathrm{~ns}$. Note that the water molecules are highly ordered, with their dipoles aligned along the direction of the electric field induced by the charge imbalance. The time at which the pore forms is dependent on the initial charge imbalance.

In Fig. 6, we show the number of water molecules in the pore region as a function of time for simulations at $500 \mathrm{mM}$ $\mathrm{NaCl}$ (top) and $0 M \mathrm{NaCl}$ (bottom). A water molecule is (somewhat arbitrarily) defined to be in the pore region if the oxygen atom is at a distance of $8 \AA$ or less from the center of the bilayer. Once formed, the pore rapidly expands to a peak value within a time span of a few hundred picoseconds. Then, the number of pore water molecules decreases as the pore shrinks. In the time scale of our simulations (10-30 ns), the pores never disappear completely. However, other authors ${ }^{6}$ have observed pore closure at $\sim 60 \mathrm{~ns}$ in a DMPC bilayer with an initial charge imbalance of $6 e$. For reasons yet entirely unclear, the pore formation is accelerated at low salt concentrations. In simulations D000-6 to D00010 , on average, the pore formation occurs earlier than in the

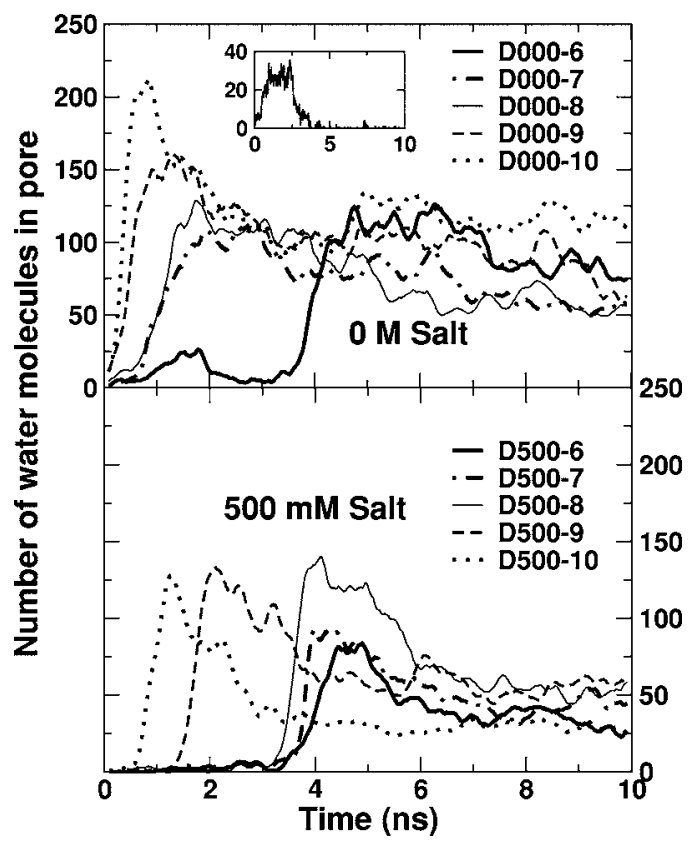

FIG. 6. The number of water molecules in the pore region as a function of time for simulations at $0 M$ salt (top) and $500 \mathrm{mM}$ salt (bottom). In most simulations, only one of the two bilayers forms a pore. The inset shows the number of water molecules in the pore region of the second bilayer of simulation D000-9, which also forms a second pore, which closes after $\sim 4$ ns. corresponding simulation at $500 \mathrm{mM}$ (D500-6 to D500-10) (Fig. 6, bottom). This is presumably because the local electrostatic fluctuations are larger at low concentrations of salt, leading to larger local electric fields. At higher concentrations of salt, the fluctuations are probably muted due to the screening effects by the other ions. Also, the peak value for the number of pore water molecules seems larger at low salt concentration than the corresponding high salt simulation, suggesting that larger local electric fields are possible at low salt concentrations. In almost all the simulations, the pore formation occurs in only one of the bilayers. However, in one of the simulations (D000-9), we observed pores in both the bilayers (inset, Fig. 6). The second pore, however, does not translocate any ions through it and closes after a short duration. While pores form within $10 \mathrm{~ns}$ for charge imbalances of $6 e$ or greater, it takes $\sim 20 \mathrm{~ns}$ for a pore to form under an initial charge imbalance of $5 e$. We do not observe any pore formation at a charge imbalance of less than $5 e$ even at $\sim 50 \mathrm{~ns}$. Thus, for this system size, a charge imbalance of $5 e$ seems to represent a threshold potential difference beyond which dielectric breakdown will occur rapidly. In an earlier study, Gurtovenko and Vattulainen ${ }^{6}$ found that DMPC lipids formed pores with a charge imbalance of $5 e$ for system sizes identical to ours albeit at time scales faster than found in this study. However, DMPC lipids have a smaller hydrophobic thickness than POPC bilayers used in this study and a slightly smaller charge imbalance may be sufficient to cause pore formation. Thus our simulations are consistent with observations from other studies.

In Fig. 7, we show the number of pore water molecules and the charge imbalance as a function of time for a representative simulation, D000-9. The abscissa on the right shows the charge imbalance, while the one on the left shows the number pore water molecules. The arrows on the curves point towards the relevant abscissa. From a peak of $\sim 170$ water molecules in the pore, the pore shrinks to $\sim 70$ water molecules after $10 \mathrm{~ns}$, in the meantime translocating seven ions (three $\mathrm{Cl}^{-}$ions and four $\mathrm{Na}^{+}$ions). In all our simulations, we observe both anions and cations translocating through the pore. In the time scale of the simulations (10-20 ns), the charge imbalance is typically reduced to $2 e$. In no simulation did we observe total discharge of the imbalance, but presumably simulations longer by at least an order of magnitude will capture this effect unless the pore closes before the imbalance is completely removed. We must men- 


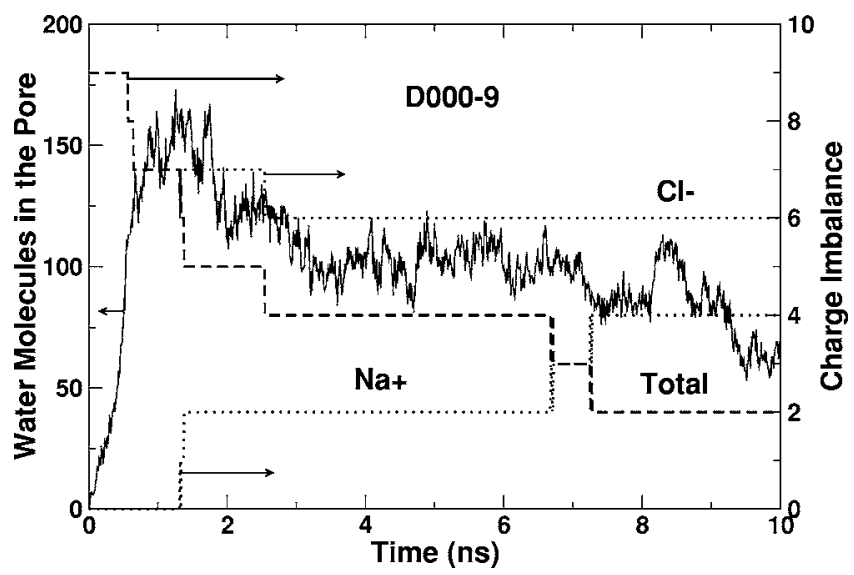

FIG. 7. The number of water molecules in the pore (thin lines, left abscissa) and the instantaneous charge imbalance (thick dashed line, right abscissa) as functions of simulation time. The number of $\mathrm{Na}^{+}$and $\mathrm{Cl}^{-}$ions in the inner water compartment is also shown as dotted lines. The arrows on the curve point towards the relevant abcissa. Four cations and three anions pass through the pore in this simulation.

tion at this point that our simulation setup is slightly different from that of Gurtovenko and Vattulainen ${ }^{6}$ in that in our simulations the charge imbalance is created by a difference in both cations and anions in both compartments. However, in their simulations, the number of anions was identical in both water compartments, while the number of cations was lower than the number of anions in one compartment and higher than the number of anions in the other (so that overall charge neutrality was still maintained). In other words, we set up the simulation at $500 \mathrm{mM}$ and +1 charge difference per bilayer by generating $500 \mathrm{mM}$ of $\mathrm{Na}^{+}$and $\mathrm{Cl}^{-}$ions in each compartment, and then adding one additional $\mathrm{Na}^{+}$ion to one compartment and one additional $\mathrm{Cl}^{-}$ion to the other compartment. However, their setup would have accomplished the imposition of the same charge difference by adding one $\mathrm{Na}^{+}$ ion to one compartment and removing one $\mathrm{Na}^{+}$ion from the other compartment. Thus, they do not have an anion gradient and thus presumably did not observe any anion translocation, while we observe both anion and cation translocations. However, even in the absence of a gradient in the anion concentration, rare instances of anion translocation can occur as seen in the simulations of an implicit hydrophobic pore by Dzubiella and Hansen. ${ }^{7}$

\section{Ion translocation}

In Fig. 8 we show the $z$ coordinates (direction normal to the bilayer) of the ions that translocate through the pore in two representative simulations (D000-7, top and D000-8, bottom). The bilayer center is at 0 (thin, dashed line) and the average positions of the phosphorus atoms of the top and bottom lipid leaflets are shown for reference (thick dashed lines). The top water compartment has an excess of $\mathrm{Cl}^{-}$ions and the bottom water compartment has an excess of $\mathrm{Na}^{+}$ ions. For D000-7, we observe two anion (thick lines) and two cation (thin lines) translocations. In most cases, the residence time for the anion inside the hydrophobic pore region is independent of the instantaneous charge imbalance. However, the residence time for the cations in the hydrophobic

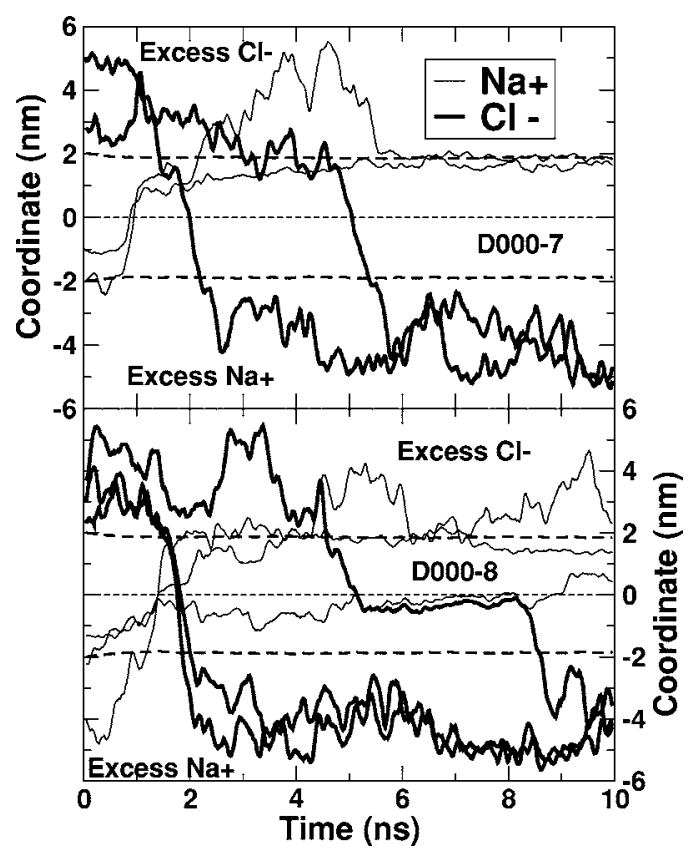

FIG. 8. The $z$ coordinates (direction normal to the bilayer) of all the translocating ions in simulations D000-7 (top) and D000-8 (bottom) as functions of time. The thick solid lines are the anions and the thin solid lines are the cations. The thin dashed line represents the bilayer center and each thick dashed line represents the average position of the lipid phosphorus atom in each leaflet.

region is variable. As mentioned earlier, some of the $\mathrm{Na}^{+}$ ions are bound to the lipid head groups, while some are freely diffusing in the water phase. We find that the free $\mathrm{Na}^{+}$ ions have a shorter residence time within the pore than do the bound $\mathrm{Na}^{+}$ions. This can be seen in Fig. 8, bottom (D000-8), where three cations translocate. One of them has a residence time in the pore of a few hundred picoseconds, the other $\sim 4 \mathrm{~ns}$, and the third has only partially translocated during the $10 \mathrm{~ns}$ simulation. Analysis of the trajectory shows that the cation with the shortest residence time is not bound to the lipids. The other two cations start the simulation bound to lipid molecules. One of them stays bound to a lipid molecule for the $10 \mathrm{~ns}$ duration of the simulation, while the other becomes unbound at $\sim 2 \mathrm{~ns}$ and translocates within $4 \mathrm{~ns}$. We also observe an interesting case of ion pairing (Fig. 8, D0008 ), where an anion and a cation moving in opposite directions in the pore stay paired for $\sim 4 \mathrm{~ns}$ in the middle of the bilayer, after which the pairing is broken.

In our simulations, the residence times of the anions in the pore region are largely independent of the instantaneous charge imbalance across the bilayer. This is presumably because the anions do not "bind" to the lipids lining the pores, as some cations do. The anions presumably freely diffuse through the pore. The residence times for the cations seem to depend on their initial binding state. In simulations of ion translocation through implicit hydrophobic pores, ${ }^{7}$ where the pore region is not atomistically treated, the residence time of cations in the pore region has been found to be a function of the instantaneous charge imbalance, which controls the driving electric field. Due to the high computational costs in our study, we have not been able to test this by running longer simulations. In our simulations, most ion transport occurs 
when the pore is relatively large, with $\sim 100$ water molecules in the pore region (within $\sim 8 \AA$ of the bilayer center). Also, we have not extended the simulations long enough to observe total discharge of the potential difference. It is indeed possible that at smaller pore radii and smaller bilayer potential difference, the anion and cation residence times can be higher than those observed in this work.

\section{Lipid flip-flop}

When a lipid molecule moves from one leaflet of the bilayer to another, it is said to have flip-flopped. Lipid flipflop is an extremely rare event in fully intact bilayers, due to the high free energy barrier involved in moving the polar head groups through the hydrophobic core. However, flipflop is an essential phenomenon during lipid fusion. ${ }^{24}$ Lipid flip-flop also occurs in the presence of bilayer-disrupting peptides. $^{25}$ In Fig. 9, in the top frame we show the trajectories of the phosphorus atoms of all the lipid molecules that are in the pore region for simulation D500-7. Clearly, the lipids that start in the top leaflet stay above the middle of the bilayer and those that start in the bottom leaflet stay below the middle of the bilayer for the duration of the simulation. This is true for almost all of the lipids in all the simulations where a pore is formed. However, we also observe rare instances of lipid flip-flop, where a lipid molecule starts at one of the bilayer leaflets and ends in the opposing leaflet. In Fig. 9, in the bottom frame, we show one such instance (D500-9). The flip-flop occurs within a few nanoseconds. The snapshots of the flip-flopping lipid, depicted as orange van der Waals spheres, are shown at $0,1,2,4$, and $10 \mathrm{~ns}$. While lipid flip-flop has been observed in all-atom simulations of vesicle self-assembly ${ }^{26}$ and in hybrid-coarse-grained simulations in the presence of external electric fields ${ }^{27}$ to the best of our knowledge this is the first observation of spontaneous flipflop in an all-atom simulation of lipid bilayers. In all of our simulations, we observed only two instances of lipid flipflop. Thus, it is difficult to comment on the generalized mechanism of lipid flip-flop. However, analysis of those two trajectories and other partially flip-flopped cases suggests that flip-flop may be directly correlated to cation transport, at least for zwitterionic lipids such as in this study. When a bound cation translocates, it might drag a lipid molecule along with it to the other lipid leaflet. It is also possible that at longer time scales, the lipids in the pore region can flipflop by a purely diffusive mechanism, unassisted by ion transport. Longer simulations, with more instances of lipid translocation, will help shed more light on the exact flip-flop mechanism.

\section{Force field dependence}

It is extremely likely that the choice of force field parameters influences the mechanism of pore formation and ion translocation. The two most commonly used force fields for atomistic lipid bilayer simulations are the CHARMM force field and the united-atom force field used by Berger et al. ${ }^{11}$ The force field by Berger et al. is largely based on the OPLS force field. These force fields have been shown to predict qualitatively different cation/lipid interactions. ${ }^{1,2,6,22}$ While the OPLS based force field predicts the "binding" of $\mathrm{Na}^{+}$ ions to the lipid molecules leading to lipid complexation, to our knowledge, no such observation has been made with the CHARMM based force fields to date. In the present manuscript (in which we have used the OPLS based force field of Berger et al.), we observe that bound $\mathrm{Na}^{+}$ions show characteristically different pore translocation profiles than unbound $\mathrm{Na}^{+}$ions. Thus, one could expect qualitatively different behaviors for other force fields. Also, the shape of the transbilayer potential seems to be dependent on the force field. In this work, and in other studies using OPLS force fields, ${ }^{2,6}$ the transbilayer potential profile shows a peak near the plane of the average lipid phosphorus atom (Fig. 2), whereas the study by Sachs et al., using a CHARMM force field, shows a peak in the middle of the bilayer core. ${ }^{1}$ The probable reason for the qualitative difference in the shape of the potential profile lies in the nature of the force fields. Since the OPLS based force field uses united-atom formulation for the lipid tail methyl groups, there is no partial charge on any of the lipid tail united atoms, leading to a flat potential profile along the entire length of the bilayer core. However, CHARMM is truly an all-atom force field, with explicit hydrogen atoms and small partial charges associated with those hydrogens, which leads to a nonuniform potential profile along the lipid tail region. Sampling errors can also lead to potential profiles that are not smooth. Since it is difficult to obtain precise potential profiles experimentally, at this juncture, it is not possible to comment on the superiority of one force field over the other. While such discrepancies are troubling, they will hopefully be resolved in the near future. Nevertheless, the value for the transmembrane potential difference is almost identical for both force fields, and is in agreement with other theoretical estimates, ${ }^{1}$ which is extremely encouraging.

In this work, we have performed simulations with charge imbalances of up to ten ions for systems with a size of 64 lipids per leaflet. Such large charge imbalances are extremely unlikely events. However, using such exaggerated transmembrane potential differences enables us to study pore formation and ion translocation in reasonable time scales ( $\sim 10 \mathrm{~ns}$ ). Also the mechanism of pore formation and ion translocation seems to be qualitatively similar for different transmembrane potentials. This justifies the large charge imbalances used in our studies. Treating pore formation as a process with a specific activation energy enables us to make estimates of the time it takes for pore formation at smaller imposed transmembrane potentials by fitting the pore formation time to the exponential of the imposed potential difference. In Fig. 10, we plot semilogarithmically the pore formation time as a function of transmembrane potential difference (solid circles) for simulations at $0 M$ salt. Note that the transmembrane potential was averaged from the beginning of a simulation to the time when the pore forms. To generate reasonable statistics, we ran four additional copies of simulations D000-5 to D000-10 with different initial random seeds. The standard error over the five independent simulations is also shown. For imbalances of $7 e$ and larger, pore formation occur in subnanosecond time scales, while for imbalances of $6 e$ and $5 e$, they happen, on an average, at 6 and $20 \mathrm{~ns}$, respectively. The dashed line is an exponential fit to 

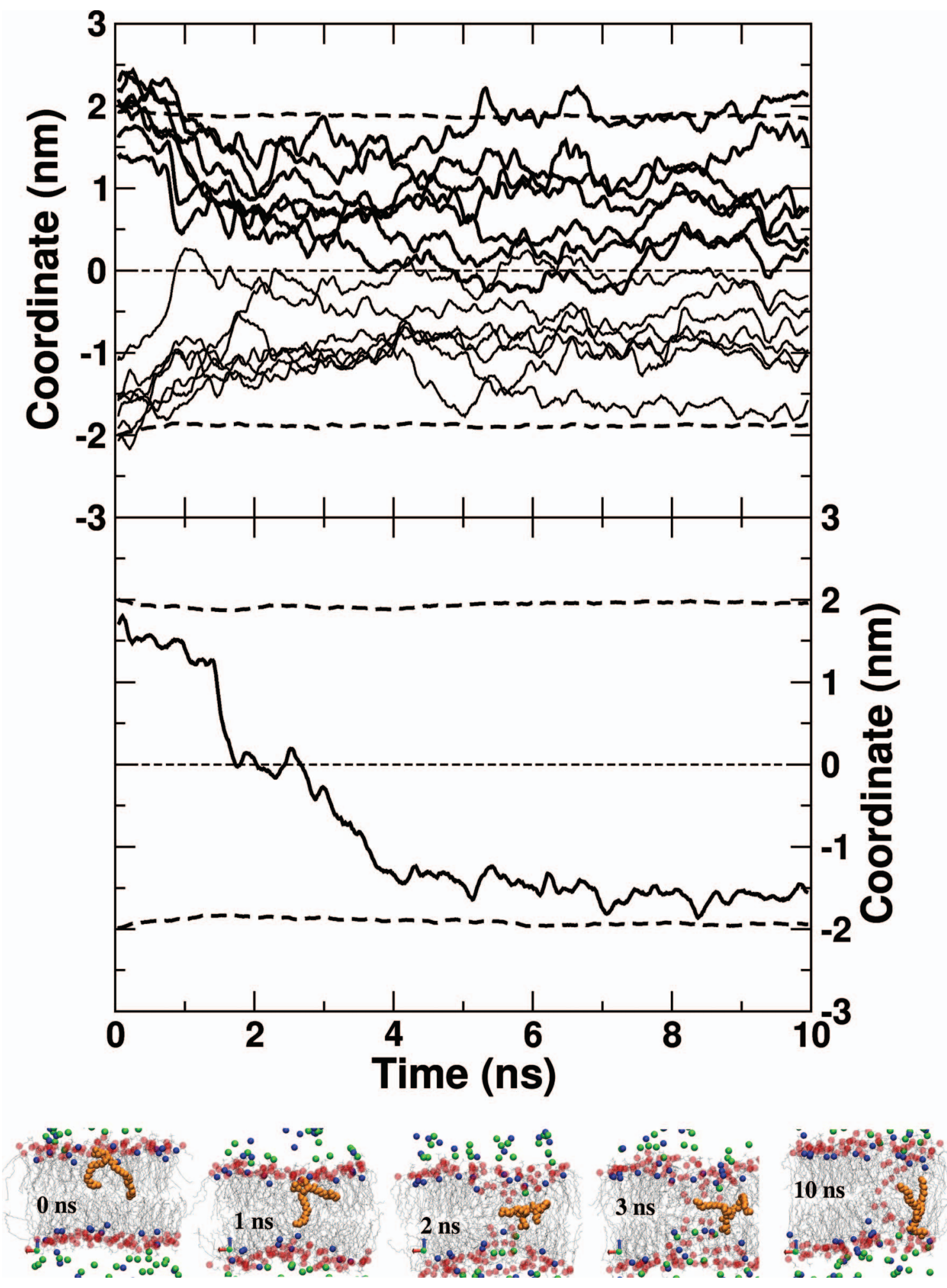

FIG. 9. (Color) Top frame: the $z$ coordinates (direction normal to the bilayer) of the lipid phosphorus atoms that line the water pore in simulation D500-7, as functions of time. The center of the bilayer and the average lipid phosphorus position are shown for reference as thin and thick dashed lines, respectively. Bottom frame: the $z$ coordinates of a flip-flopping lipid from simulation D500-8. Bottom: the snapshots of the bilayer at 0, 1, 2, 3, and 10 ns of the simulation D500-8. The flip-flopping lipid is shown as orange van der Waals spheres. The lipid starts in the top leaflet and ends in the bottom leaflet. $\mathrm{Na}^{+}$and $\mathrm{Cl}^{-}$ions are red and green spheres, respectively.

the six data points, extrapolated to smaller values of transmembrane potential differences. This should give an approximate estimate of the time for pore formation at smaller transmembrane potential differences. At $5 e$ charge imbalance, which represents the threshold charge imbalance, only four of the five simulations formed pores in under $30 \mathrm{~ns}$. Thus, the standard error for that potential difference is averaged over four data points. The estimates obtained from our simulations are approximate and should be treated with caution. Figure 10 predicts that, if a single scaling regime is 


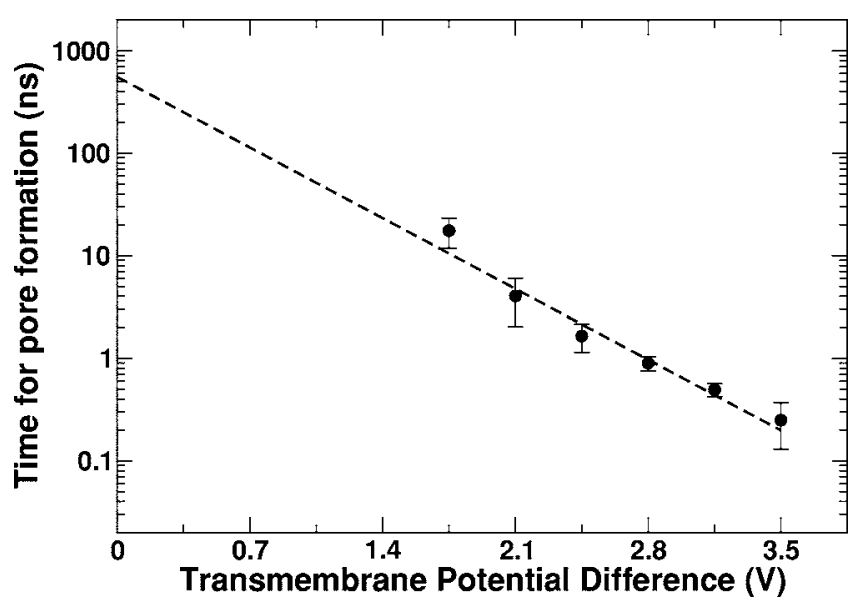

FIG. 10. Semilog plot of pore formation time as a function of transmembrane potential difference.

assumed, spontaneous pore formation (at zero potential difference) occurs in microsecond time scale. This is clearly an overestimation by at least a few orders of magnitude. Running more simulations at each charge imbalance and obtaining an average pore formation time should yield better estimates for pore formation times at lower transmembrane potential differences. Longer simulations should also enable us to obtain better estimates for pore formation times at lower transmembrane potentials and a better understanding of the scaling behavior. From our simulations, especially those at higher salt concentration, it is clear that a reasonably large water layer is needed to avoid artifacts. From Fig. 3, it is clear that water is ordered for distances of at least $\sim 1.5 \mathrm{~nm}$ from the plane of the lipid phosphorus atoms and so the thickness of the water compartments should be chosen carefully.

\section{CONCLUSIONS}

We have performed all-atom simulations of a double bilayer setup, systematically changing the potential difference across the bilayers by explicitly varying the number of anions and cations in the two water compartments. For charge imbalances as large as $4 e$ per bilayer, the bilayers are stable for times up to $50 \mathrm{~ns}$ and show a monotonic increase in transbilayer potential difference. At charge imbalances greater than $4 e$, dielectric breakdown occurs, leading to the formation of water pores in the bilayer. Anions and cations translocate across the bilayer through the pore leading to reduction in the transmembrane potential difference. In the time scale of the simulations (10-30 ns), we do not observe a full potential discharge, and typically there is a residual charge imbalance of $2 e$ at the end of the simulations. As the ions translocate, the size of the pore decreases, but the pores do not fully close, remaining stable for the entire duration of the simulations $(10-30 \mathrm{~ns})$. For a given salt concentration, the time necessary for pore formation is dependent on the charge imbalance, with quicker pore formation at higher ion imbalances. For a given charge imbalance, the pore formation is faster at lower salt concentrations. In general, the anions seem to have a uniform residence time in the pore region, independent of the instantaneous charge imbalance. However, the cation residence time seems to be dependent on whether the cation $\left(\mathrm{Na}^{+}\right)$is bound to a lipid molecule or not. We also observe instances of lipid flip-flop, where lipid molecules move from one bilayer leaflet to another, assisted by the water pore. It is clear that the double bilayer setup, which enables an explicit tuning of transmembrane potential difference, is an excellent model to study membraneassociated processes where such potential differences are critical. Not only is the model ideal to study processes such as transport through ion channels and other membrane proteins but also to transport of molecules through transient hydrophilic pores in lipid bilayers.

${ }^{1}$ J. N. Sachs, P. S. Crozien, and T. B. Woolf, J. Chem. Phys. 121, 10847 (2004).

${ }^{2}$ A. A. Gurtovenko, J. Chem. Phys. 122, 244902 (2005).

${ }^{3}$ R. J. Clarke, Adv. Colloid Interface Sci. 89-90, 263 (2001).

${ }^{4}$ A. Ottova and H. T. Tien, Bioelectrochemistry 56, 171 (2002).

${ }^{5}$ D. P. Tieleman, BMC Biochem. 5, 10 (2004).

${ }^{6}$ A. A. Gurtovenko and I. Vattulainen, J. Am. Chem. Soc. 127, 17570 (2005).

${ }^{7}$ J. Dzubiella and J. P. Hansen, J. Chem. Phys. 122, 234706 (2005).

${ }^{8}$ J. Dzubiella, R. J. Allen, and J. P. Hansen, J. Chem. Phys. 120, 5001 (2004).

${ }^{9}$ E. Lindahl, B. Hess, and D. van der Spoel, J. Mol. Model. 7, 306 (2001).

${ }^{10}$ D. Van Der Spoel, E. Lindahl, B. Hess, G. Groenhof, A. E. Mark, and H. J. Berendsen, J. Comput. Chem. 26, 1701 (2005).

${ }^{11}$ O. Berger, O. Edholm, and F. Jahnig, Biophys. J. 72, 2002 (1997).

${ }^{12}$ D. P. Tieleman, H. J. C. Berendsen, and M. S. P. Sansom, Biophys. J. 80, 331 (2001).

${ }^{13}$ D. P. Tieleman, H. Leontiadou, A. E. Mark, and S. J. Marrink, J. Am. Chem. Soc. 125, 6382 (2003).

${ }^{14}$ S. J. Marrink, E. Lindahl, O. Edholm, and A. E. Mark, J. Am. Chem. Soc. 123, 8638 (2001).

${ }^{15}$ M. Patra and M. Karttunen, J. Comput. Chem. 25, 678 (2004).

${ }^{16}$ M. Patra, M. Karttunen, M. T. Hyvonen, E. Falck, P. Lindqvist, and I. Vattulainen, Biophys. J. 84, 3636 (2003).

${ }^{17}$ U. Essmann, L. Perera, M. L. Berkowitz, T. Darden, H. Lee, and L. G. Pedersen, J. Chem. Phys. 103, 8577 (1995).

${ }^{18}$ B. Hess, H. Bekker, H. J. C. Berendsen, and J. G. E. M. Fraaije, J. Comput. Chem. 18, 1463 (1997).

${ }^{19}$ S. A. Pandit and M. L. Berkowitz, Biophys. J. 82, 1818 (2002).

${ }^{20}$ S. A. Pandit, D. Bostick, and M. L. Berkowitz, Biophys. J. 84, 3743 (2003).

${ }^{21}$ J. N. Sachs and T. B. Woolf, J. Am. Chem. Soc. 125, 8742 (2003).

${ }^{22}$ R. A. Bockmann, A. Hac, T. Heimburg, and H. Grubmuller, Biophys. J. 85, 1647 (2003).

${ }^{23}$ J. N. Sachs, H. Nanda, H. I. Petrache, and T. B. Woolf, Biophys. J. 86, 3772 (2004).

${ }^{24}$ W. C. Lin, C. D. Blanchette, T. V. Ratto, and M. L. Longo, Biophys. J. 90, 228 (2006).

${ }^{25}$ K. Matsuzaki, O. Murase, N. Fujii, and K. Miyajima, Biochemistry 35, 11361 (1996)

${ }^{26}$ A. H. de Vries, A. E. Mark, and S. J. Marrink, J. Am. Chem. Soc. 126, 4488 (2004).

${ }^{27}$ Q. Hu, R. P. Joshi, and K. H. Schoenbach, Phys. Rev. E 72, 031902 (2005). 